\title{
Développement de sapinières éclaircies exposées à une épidémie de tordeuse des bourgeons de l'épinette
}

\author{
par David Pothier ${ }^{1}$
}

En 1968, trois intensités d'éclaircie par le bas ont été appliquées dans des sapinières denses de l'Est du Québec âgées de 33 ans de façon à enlever 0,20 et $30 \%$ de la surface terrière totale. Les aires d'étude étaient situées dans deux secteurs distincts dont l'un a bénéficié d'arrosages aériens annuels d'insecticides entre 1978 et 1982 alors que l'autre n'a pas été protégé contre une épidémie de tordeuse de bourgeons de l'épinette (TBÉ) (Choristineura fumiferana [Clem.]) qui s'est manifestée de 1976 à 1983. Des mesures dendrométriques ont été périodiquement effectuées entre 1968 et 1994 dans 32 placettes échantillons de 0,04 ha. Les éclaircies par le bas ont surtout éliminé des arbres non marchands (DHP $<9,1$ $\mathrm{cm}$ ), ce qui a laissé de 5000 à 7000 tiges/ha alors que la densité des placettes témoins atteignait 10000 tiges/ha. La production annuelle nette en volume marchand était d'environ $8 \mathrm{~m}^{3} / \mathrm{ha}$ entre 1968 et 1976 sans qu'il n'y ait de différence marquée entre les niveaux d'éclaircie. Pendant la période de défoliation sévère due à la TBÉ (1976-1985), la production annuelle nette en volume marchand des placettes ayant bénéficié d'arrosages d'insecticides a diminué de moitié mais a repris son niveau d'avant l'épidémie à partir de 1985. De leur côté, les placettes non protégées contre la TBÉ ont eu une production nette fortement négative pendant la période épidémique et cette mortalité s'est poursuivie par la suite jusqu'à la perte de tous les arbres. Si les éclaircies n'ont pratiquement pas affecté la production en volume marchand des placettes pendant la période de 26 ans qu'a duré l'étude, l'accroissement annuel moyen en DHP, pour sa part, a été inversement proportionnel à la densité des peuplements. Cet avantage des placettes éclaircies aurait pu être plus accentué si elles avaient fait l'objet d'un prélèvement plus élevé au commencement de l'étude. En effet, l'analyse de l'évolution de la densité des peuplements en fonction du volume moyen des arbres montre que le taux de mortalité des arbres de l'ensemble des placettes est semblable à celui décrit par la relation d'auto-éclaircie, ce qui suggère que même les arbres des placettes les plus éclaircies ont dû subir une forte compétition intraspécifique.

Mots clés : sapin baumier, éclaircie par le bas, tordeuse des bourgeons de l'épinette, production en volume marchand, accroissement en diamètre, relation d'auto-éclaircie
In 1968, three intensities of low thinning were applied in $33-$ year-old balsam fir (Abies balsamea [L.] Mill.) stands of eastern Québec in order to remove 0,20 and $30 \%$ of the total basal area. Sample plots were located in two distinct areas, one of which was annually sprayed with insecticides between 1978 and 1982 while the other was never protected against a spruce budworm (Choristoneura fumiferana [Clem.]) outbreak that occurred between 1976 and 1983. A total of 32 0.04-ha sample plots were periodically measured between 1968 and 1994. Low thinnings mostly removed non merchantable trees $(\mathrm{DBH}<9.1 \mathrm{~cm})$ and left 5000 to 7000 trees/ha while stand density of control plots reached 10000 stems/ha. From 1968 to 1976, net annual increment in merchantable volume was around $8 \mathrm{~m}^{3} /$ ha but no differences were detected between thinning levels. During the period of severe defoliation caused by spruce budworm (1976-1985), net annual increment in merchantable volume of sample plots sprayed with insecticides decreased by $50 \%$ but get back to its initial level after 1985. Net annual increment of sample plots that were never protected against spruce budworm was strongly negative during the outbreak period until the extinction of all trees was completed. While thinning level only slightly affected merchantable yield of sample plots during the 26-year period, mean annual increment in DBH was inversely proportional to stand density. This advantage of thinned plots could have been greater if stand density had been reduced further at the beginning of the study. Indeed, the analysis of the evolution of stand density as a function of mean volume of trees shows that mortality rate of all sample plots was similar to that given by the self-thinning rule. This suggests that even trees of most heavily thinned plots suffered from strong intra-specific competition.

Key words: balsam fir, low thinning, spruce budworm, growth and yield, diameter increment, self-thinning rule

\section{Introduction}

En diminuant la densité des peuplements, les coupes d'éclaircie modifient les conditions environnementales des arbres résiduels, ce qui améliore généralement leur croissance. Cette meilleure croissance peut être liée à une hausse de la disponibilité en eau du sol puisque l'éclaircie réduit l'interception des précipitations et l'évapotranspiration potentielle (Aussenac et al. 1982; Whitehead et al. 1984; Aussenac et Granier 1988). Cependant, une augmentation de la disponibilité de la lumière semble être le facteur le plus important pour expliquer l'impact d'une diminution de la compétition sur la croissance des

${ }^{1}$ Direction de la recherche forestière, Ministère des Ressources naturelles du Québec, 2700, rue Einstein, Sainte-Foy (Québec), G1P 3W8. arbres (Brand 1986). À court terme, la plus grande quantité de lumière disponible pour les feuilles a pour effet d'augmenter leur taux de photosynthèse et, par conséquent, la quantité de bois produite annuellement par unité de superficie foliaire (Brix 1983; Pothier et Margolis 1991). Toutefois, à plus long terme, la meilleure croissance des arbres éclaircis semble davantage liée à une hausse de leur biomasse foliaire (Brix 1983).

L'augmentation de l'accroissement diamétral des arbres est souvent évoquée pour justifier l'application d'une éclaircie. Cependant, d'autres arguments d'ordre sylvicole ou économique militent en faveur de la coupe d'éclaircie. Ainsi, la possibilité de sélectionner les meilleurs sujets, la production de revenus intermédiaires et la réduction de la durée de la révolution par l'atteinte rapide d'arbres de dimensions marchan- 
des sont tous des avantages associés à l'éclaircie (Pardé 1964; Bailly 1992; Guitton et Ruchaud 1996). L'éclaircie peut provoquer l'établissement d'une strate de régénération bien développée sous le couvert d'un peuplement (Hatcher 1961; Corriveau 1971), ce qui constitue un avantage dans le cas d'un aménagement axé sur la régénération naturelle. Des gains sur le plan de la biodiversité peuvent aussi être produits par une éclaircie. Par exemple, Bailly (1992) et Guitton et Ruchaud (1996) ont noté une hausse de l'abondance et de la diversité faunique et floristique dans des peuplements éclaircis à la suite du développement d'une strate arbustive propice à la présence d'animaux phytophages et à la nidification de plusieurs espèces d'oiseaux. De plus, l'augmentation de l'intensité lumineuse au sol peut résulter en un réchauffement des horizons superficiels qui peut stimuler l'activité biologique du sol et le recyclage des éléments minéraux (Piene 1978; Bailly 1992; Guitton et Ruchaud 1996).

La récolte des arbres destinés à mourir en absence d'intervention pourrait potentiellement accroître la production marchande totale d'un peuplement (Reukema et Bruce 1977). Cependant, les éclaircies prélèvent généralement davantage d'arbres que ceux qui sont sujets à mourir dans un avenir rapproché de sorte que le peuplement résiduel ne peut pleinement profiter de l'espace inoccupé, ce qui réduit le gain en volume escompté de la récupération de la mortalité (Reukema et Bruce 1977; Hamilton 1981). Malgré tout, l'objectif de récolte des arbres destinés à mourir est souvent atteint au cours des opérations d'éclaircie comme l'indiquent des taux de mortalité proportionnels à la surface terrière résiduelle $(c f$. Bertrand et Bolghari 1970; Weetman 1975; Stiell 1980; Weetman et al. 1980; Harrington et Reukema 1983). Outre la récupération des arbres voués à mourir dans de courts délais, l'éclaircie peut aussi réduire l'incidence de la mortalité sur les arbres résiduels en augmentant leur vigueur et, par conséquent, en les rendant moins vulnérable aux attaques par les insectes (Mitchell et al. 1983; Coyea et Margolis 1994; Thibault et al. 1995). Toutefois, les éclaircies peuvent aussi avoir des effets adverses sur le taux de survie des peuplements. Ainsi, Hatcher (1961) et Corriveau (1971) ont observé qu'une diminution trop forte de la densité de sapinières pouvait augmenter la susceptibilité des arbres à être renversés par le vent.

La présente étude a été initiée en 1968 dans le but de documenter l'accroissement, la production et le taux de mortalité d'une jeune sapinière à des éclaircies par le bas. Ce type de peuplement étant fréquemment rencontré dans l'Est du Québec, l'étude visait ainsi à contribuer à la bonification de l'aménagement des forêts de seconde venue de cette partie du Québec. Cependant, la crainte de provoquer des chablis en intervenant dans ce type de peuplement a limité l'intensité des éclaircies à des niveaux relativement faibles. De plus, l'arrivée d'une épidémie de tordeuse des bourgeons de l'épinette au milieu des années 1970 a permis de déterminer son impact sur la croissance du peuplement, de caractériser l'effet des éclaircies sur la vulnérabilité du peuplement et de vérifier l'efficacité de pulvérisations aériennes d'insecticides chimiques.

\section{Matériel et méthodes \\ Secteur d'étude}

Le secteur d'étude est situé au Québec près du lac Matapédia ( $48^{\circ} 34^{\prime}$ de latitude $\mathrm{N}$.; $67^{\circ} 32^{\prime}$ de longitude $\mathrm{O}$.) à une altitude variant de 200 à $250 \mathrm{~m}$. Les peuplements sont établis sur des tills loameux modérément bien drainés dont la profondeur moyenne est de $45 \mathrm{~cm}$ (Thibault et al. 1995). Ils sont composés à plus de $98 \%$ de sapin baumier (Abies balsamea [L.] Mill.) qui est accompagné de quelques épinettes blanches (Picea glau$c a$ [Moench] Voss) et bouleaux à papier (Betula papyrifera Marsh.). Les strates muscinale et herbacée sont respectivement dominées par Hylocomium splendens (Hedw.) BSG. et Oxalis montana Raf. et l'indice de qualité de station est de $14 \mathrm{~m}$ à 50 ans. Les peuplements sont issus d'une coupe à blanc exécutée au cours des années 1930 et des études d'arbres ont démontré qu'ils étaient âgés d'environ 33 ans en 1968.

\section{Traitements}

En 1968, trois intensités d'éclaircie, enlevant respectivement 0,20 et $30 \%$ de la surface terrière totale initiale, ont été appliquées sur des superficies expérimentales de $30 \times 30 \mathrm{~m}$. Les éclaircies ont été limitées à ces pourcentages de prélèvement parce que les risques de chablis étaient jugés trop importants si davantage d'arbres avaient été éliminés. Les différentes intensités d'éclaircie ont été respectées en exécutant un martelage qui visait à éliminer principalement les arbres de faibles dimensions tout en uniformisant le prélèvement à travers les blocs traités. Il s'agissait donc d'une éclaircie par le bas qui n'avait pas pour objectif de récolter de volume marchand. La prise de données dendrométriques a été réalisée dans des placettes de 0,04 ha établies au centre des superficies traitées. L'application des traitements a produit une gamme de surfaces terrières résiduelles qui ont été regroupées en trois classes pour faciliter la présentation des résultats (tableau 1). Ainsi, un total de 32 placettes a été établi pour l'ensemble du dispositif expérimental. De ce nombre, 23 placettes localisées dans un secteur d'étude ont reçu des arrosages aériens annuels d'insecticides (aminocarb ou fenitrothion) entre 1978 et 1982 inclusivement de façon à combattre une épidémie de tordeuse des bourgeons de l'épinette (TBÉ) (Choristoneura fumiferana (Clem.)). Les neuf autres placettes (trois par classe de surface terrière résiduelle), regroupées dans un autre secteur, n'ont reçu aucun arrosage d'insecticide pendant toute la durée de l'étude et ont donc permis de suivre l'évolution de ces sapinières en absence de protection contre la TBÉ. Les traitements ont été aléatoirement distribués à l'intérieur de chacun de ces deux secteurs.

\section{Inventaires}

Avant la coupe, chaque placette de 0,04 ha a été délimitée de façon à pouvoir dénombrer les arbres par espèce et par classe de DHP de 2,54 cm. Après la coupe, les arbres restants de chaque placette ont été numérotés et leur DHP a été mesuré au millimètre près à l'aide d'un ruban diamétrique. De plus, une dizaine d'arbres par placette ont été sélectionnés pour établir des relations DHP-hauteur. Il était initialement prévu de reprendre cet inventaire à tous les cinq ans mais l'apparition d'une épidémie de TBÉ en 1974 a donné l'occasion d'intensifier les périodes de mesurage dans le but de documenter les effets de l'épidémie sur la production des sapinières. Ainsi, outre l'inventaire initial de 1968, chaque placette a été inventoriée de nouveau en 1973, 1976, 1977, 1978, 1979, 1980, 1981, 1983, 1985, 1989 et 1994.

\section{Analyse des données}

À partir des inventaires, il a été possible de calculer le nombre total de tiges à l'hectare ainsi que le nombre de tiges marchan- 
Tableau 1. Caractéristiques des peuplements en 1968 avant et après l'application des traitements

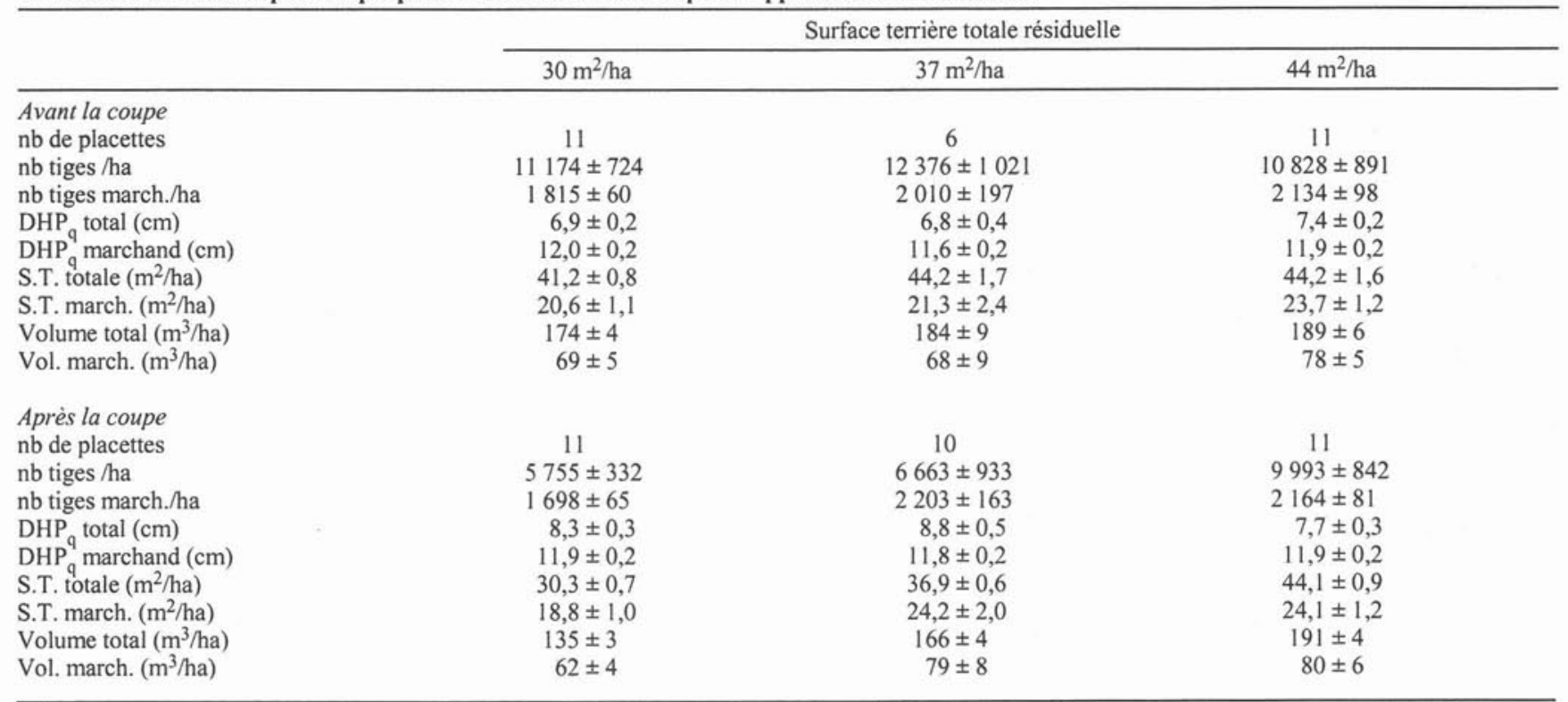

Note : Chaque moyenne ( \pm erreur type) a été calculée à partir du nombre de placettes indiqué pour chaque classe de surface terrière. Les données avant la coupe de quatre placettes de la classe de surface terrière résiduelle de $37 \mathrm{~m}^{2} / \mathrm{ha}$ n'ont pu être retrouvées dans les archives, ce qui peut expliquer certains écarts entre les valeurs d'avant et d'après la coupe.

des (DHP $\geq 9,1 \mathrm{~cm})$. Il a également été possible de calculer les surfaces terrières totale et marchande et d'en déduire les DHP quadratiques total et marchand. Le volume total a été calculé en utilisant le tarif de cubage de Honer et al. (1983) qui relie le volume total d'un arbre à son DHP et à sa hauteur. Puisque la hauteur n'a pas été mesurée pour chaque arbre, une équation de régression linéaire reliant la hauteur au DHP et au $(\mathrm{DHP})^{2}$ a été calculée pour chaque année d'inventaire où ces données étaient disponibles. L'examen des résidus de ces équations de régression a révélé que le modèle utilisé s'ajustait bien aux données et que les coefficients de détermination $\left(\mathrm{R}^{2}\right)$ se situaient généralement au-delà de $70 \%$. Ces équations de régression ont ensuite été substituées dans le tarif de cubage général de Honer et al. (1983). Le calcul du volume marchand s'est effectué de la même façon en utilisant cependant le tarif de cubage marchand brut de Perron (1985).

Puisque les arbres de chacune des placettes ont été numérotés, il a été possible de calculer et de décomposer l'accroissement en volume marchand selon les définitions de Davis et Johnson (1987) et Rondeux (1993). Ainsi, l'accroissement net correspond à la différence du volume des arbres vivants entre deux inventaires en excluant le recrutement; l'accroissement des recrues correspond au volume des arbres de moins de 9,1 cm au DHP au moment d'un inventaire mais qui avaient atteint ce diamètre lors de l'inventaire suivant; et l'accroissement de la mortalité correspond au volume des arbres morts entre deux inventaires. La production nette des placettes a été déterminée en calculant la différence du volume final et du volume initial d'une période donnée en incluant le volume des recrues (Rondeux 1993).

De façon à pouvoir mettre en relation l'accroissement en diamètre des sapins en fonction de la surface terrière résiduelle totale de 1968, il a fallu calculer un accroissement en diamètre moyen pour chaque placette pour la période de 1968 à 1994. De façon à éviter d'inclure dans ce calcul un grand nombre d'arbres opprimés, cet accroissement a été calculé à partir des 800 plus grosses tiges à l'hectare parce qu'elles constituent généralement une très forte proportion du volume récolté à maturité. Puisque les placettes échantillons ont une superficie de $400 \mathrm{~m}^{2}$, l'accroissement moyen en DHP pour la période de 1968 à 1994 a donc été calculé à partir des observations faites sur les 32 plus gros arbres par placette déterminés selon leur DHP de 1994.

L'évolution du nombre de tiges à l'hectare en fonction du volume moyen par tige a été représentée graphiquement à l'aide d'un diagramme de gestion de la densité des peuplements qui comprend des informations de référence suggérées par Drew et Flewelling (1979). Le fondement de ce diagramme repose sur la relation de l'auto-éclaircie qui exprime le lien mathématique entre le volume moyen maximal qu'un arbre peut atteindre $\left[\bar{v}\left(\mathrm{dm}^{3}\right)\right]$ et le nombre de tiges à l'hectare $(\mathrm{N})$. Cette relation a été calculée par Tremblay et al. (1997) pour le sapin baumier à partir de placettes n'ayant pas été affectées par des perturbations majeures comme la TBÉ. Selon cette étude, la relation d'auto-éclaircie pour le sapin baumier peut se formuler de la façon suivante :

$$
\bar{v}=10^{7,201} N^{-1,466}
$$

Pour des sapinières denses de structure équienne, l'équation [1] décrit le taux de mortalité provoqué par la compétition intraspécifique. Drew et Flewelling (1979) ont défini une zone de densité où la mortalité est imminente à cause de la trop forte compétition. Cette zone est délimitée par la relation d'auto-éclaircie à l'intérieur de laquelle la notion de densité relative $\left(\rho_{\mathrm{r}}\right)$ est introduite:

$$
\rho_{r}=\frac{N_{0}}{N}
$$


La densité relative est définie comme étant le rapport de la densité observée d'un peuplement $\left(\mathrm{N}_{0}\right)$ et de la densité maximale que ce peuplement peut atteindre $(\mathrm{N})$ pour un même volume moyen des arbres. L'équation [1] peut donc se reformuler:

$$
\bar{v}=10^{7,201}\left(\frac{N_{0}}{\rho_{r}}\right)^{-1,466}
$$

Ainsi, la limite supérieure de la zone de mortalité imminente induite par la compétition est définie par l'équation [3] lorsqu'une densité relative égale à un (1) y est appliquée. La limite inférieure de cette zone de mortalité a été fixée à une valeur $\rho_{\mathrm{r}}=0,55$ qui correspond au seuil d'un brusque changement du taux de mortalité du sapin baumier (Tremblay et al. 1997).

Pour faciliter l'utilisation du diagramme de gestion de la densité des peuplements, Drew et Flewelling (1979) ont suggéré de tracer des isolignes du diamètre quadratique moyen ( $\left.\mathrm{DHP}_{\mathrm{q}}\right)$ et de la hauteur dominante $\left(\mathrm{H}_{\mathrm{d}}\right)$. Quoiqu'il aurait été possible d'utiliser les relations calculées par Tremblay et al. (1997) à partir de plusieurs sapinières du Québec, des paramètres plus ajustés au DHP des arbres de la présente étude ont été calculés à partir d'une forme d'équation suggérée par Newton et Weetman (1993):

$$
L O G_{10}(\bar{v})=b_{0}+b_{1} L O G_{10}(N)+b_{2} L O G_{10}\left(D H P_{q}\right)
$$

Cette régression a été calculée à partir des données des 12 années de mesure des 23 placettes qui ont été protégées contre la TBÉ $(n=276)$. Les coefficients de régression et les statistiques associées sont les suivants: $b_{0}=-0,3560, b_{1}=-$ $0,1003, b_{2}=2,3252$, le coefficient de détermination $\left(R^{2}\right)=0,992$ et l'erreur type de l'estimé $=0,0201$.

Par ailleurs, les isolignes de hauteur dominante $\left(\mathrm{H}_{\mathrm{d}}\right)$ des peuplements ont pu être calculées en déterminant la $\mathrm{H}_{d}$ de chaque placette pour chaque période d'inventaire. Pour déterminer la hauteur dominante, les quatre plus gros arbres de chaque placette [correspondant aux 100 plus gros arbres à l'hectare (Pardé et Bouchon 1988)] ont été sélectionnés en se basant sur leur DHP. Le DHP moyen de ces quatre plus gros arbres par placette a ensuite été calculé et introduit dans les équations de régression reliant la hauteur au DHP et au $(\mathrm{DHP})^{2}$. Les $\mathrm{H}_{\mathrm{d}}$ ainsi calculées ont été introduites dans le modèle:

$$
L O G_{10}(\bar{v})=b_{3}+b_{4} L O G_{10}(N)+b_{5} L O G_{10}\left(H_{d}\right)
$$

Les coefficients de régression et les statistiques associées à l'équation [5] sont les suivants: $b_{3}=2,3010, b_{4}=-0,7384, b_{5}=1,8632$, $\mathrm{R}^{2}=0,961$ et l'erreur type de l'estimé $=0,0432$.

\section{Analyses statistiques}

Pour détecter des écarts statistiquement significatifs entre les trois regroupements de surface terrière résiduelle avant l'application des traitements, les valeurs de volume marchand ont été soumises à une analyse de variance selon un dispositif complètement aléatoire. Les résultats de ce test n'indiquant aucune différence significative $(\alpha=0,05)$, les volumes marchands mesurés après l'application des traitements ont été soumis à une analyse de variance à mesures répétées (Potvin et al. 1990) qui n'a pas non plus permis de détecter de différence significative entre les trois niveaux de surface terrière résiduelle. Finalement, les trois composantes de l'accroissement (net, recrues et mortalité) ont été soumises à des analyses de variance selon un dispositif complètement aléatoire pour chacune des trois périodes définies en fonction de l'épidémie de TBÉ.

\section{Résultats et discussion}

En 1968, avant l'application des traitements, les peuplements avaient des densités dépassant 10000 tiges à l'hectare dont au moins 8000 avaient un DHP inférieur à $9,1 \mathrm{~cm}$ (tableau 1). Associées à des surfaces terrières dépassant les $40 \mathrm{~m}^{2} / \mathrm{ha}$, ces densités sont supérieures aux valeurs qu'on trouve dans les tables de Vézina et Linteau (1968) qui représentent pourtant des sapinières dont la capacité d'occupation du territoire est optimale. Les éclaircies pratiquées dans ces peuplements ont surtout éliminé les tiges non marchandes $(<9,1 \mathrm{~cm}$ au DHP) pour ne garder que 5000 à 7000 tiges à l'hectare (tableau 1), ce qui se rapproche des valeurs publiées par Vézina et Linteau (1968) pour des sapinières d'âge et de qualité de station comparables. Les éclaircies ont donc eu pour conséquence immédiate d'affecter les caractéristiques dendrométriques de l'ensemble des tiges des peuplements alors que les valeurs uniquement associées aux tiges marchandes n'ont à peu près pas été modifiées (tableau 1). Ces valeurs confirment que ce sont des éclaircies essentiellement par le bas qui ont été exécutées, qu'elles ne visaient pas à prélever de volume marchand et qu'il n'y avait pas de sentier de débardage dans les placettes expérimentales.

Pour illustrer l'évolution du volume marchand en fonction du niveau d'éclaircie, les placettes protégées et non protégées contre la TBÉ ont été séparées parce que l'épidémie a profondément affecté le comportement de ces deux groupes de placettes (figure 1). De 1968 à 1978, l'évolution du volume marchand des peuplements protégés et non protégés contre la TBÉ a été semblable (figure 1). A partir de 1979, cependant, l'évolution du volume marchand des placettes non protégées contre la TBÉ a amorcé un déclin qui s'est poursuivi jusqu'à la mort de tous les arbres qui est survenue entre 1989 et 1994 (figure 1A). La mortalité annuelle a approché $15 \mathrm{~m}^{3} / \mathrm{ha}$ pendant la période s'étalant de 1976 à 1985 (figure 2A) alors que des relevés aériens de défoliation de ce secteur ont révélé que l'épidémie de TBÉ a été sévère de 1976 à 1983 (Thibault et al. 1995). Le taux de mortalité des sapins a donc commencé à être important après trois années consécutives de défoliation sévère, ce qui avait déjà été observé par MacLean (1980). De plus, comme l'avaient déjà constaté MacLean (1980) et Piene (1989), la baisse anticipée de mortalité des placettes éclaircies n'ayant pas bénéficié de pulvérisations d'insecticides ne s'est pas concrétisée à cause de la sévérité de l'épidémie de TBÉ. Cette mortalité survient donc malgré la hausse de vigueur des arbres et la réduction de leur vulnérabilité aux insectes qui sont censées caractériser les peuplements éclaircis (Mitchell et al. 1983; Coyea et Margolis 1994).

Dans le cas des placettes protégées contre la TBÉ, l'accroissement du volume marchand a subi un ralentissement important entre 1976 et 1985 mais une reprise de croissance s'est manifestée par la suite (figure 1B). Il est d'ailleurs possible de séparer l'évolution de ces peuplements en trois périodes délimitées en fonction de l'apparition de l'épidémie de TBÉ. La période pré-épidémique (1968-1976) est caractérisée par 
Figure 1. Évolution du volume marchand des placettes regroupées en trois classes de surface terrière résiduelle pour un secteur non protégé contre la TBÉ (A) et un autre ayant bénéficié de pulvérisations aériennes annuelles d'insecticides entre 1978 et 1982 (B).

une production annuelle en volume marchand d'environ $8 \mathrm{~m}^{3} / \mathrm{ha}$ dont près du quart est attribuable aux recrues, c'est-à-dire les arbres ayant atteint un DHP de $9,1 \mathrm{~cm}$ pendant cette période (figure 2). La mortalité en volume marchand y a été très faible et aucun écart significatif n'a été décelé entre les trois niveaux de surface terrière résiduelle. De plus, l'épidémie de TBÉ n'ayant pas encore débuté, des valeurs d'accroissement similaires sont observées dans les placettes destinées à être protégées contre la TBÉ et celles qui ne le sont pas (figure 2).

Des écarts de production nette entre les placettes protégées et non protégées contre la TBÉ sont apparents pendant la période épidémique (1976-1985) à cause de la forte mortalité survenue dans le secteur n'ayant pas profité de pulvérisations aériennes d'insecticides (figure 2). Même si la mortalité annuelle a approché $2 \mathrm{~m}^{3} / \mathrm{h}$ dans les placettes protégées contre la TBÉ, la production annuelle nette a malgré tout atteint $3 \mathrm{~m}^{3} / \mathrm{ha}$ pendant cette période sans qu'il n'y ait toutefois de différences importantes entre les niveaux de surface terrière résiduelle (figure 2B). Le faible nombre de recrues pendant cette période indique que la mortalité a beaucoup affecté les arbres de petites dimensions comme l'ont d'ailleurs constaté Beaulieu et Hardy (1982), Thibault et al. (1995) et MacLean et Piene (1995). Par conséquent, la production nette positive des placettes protégées contre la TBÉ est surtout le résultat de la croissance diamétrale des arbres appartenant à l'étage des dominants et des codominants qui ont pu conserver une certaine vigueur grâce aux pulvérisations d'insecticides.

Pendant la période post-épidémique (1985-1994), la production annuelle nette en volume marchand des placettes protégées contre la TBÉ a été semblable à celle de la période pré-épidémique malgré une mortalité plus importante (figure 2B). Il semble donc que la mortalité continue d'affecter les arbres des étages dominés au cours des années qui suivent une épidémie sévère de TBÉ, ce qui est conforme aux observations de MacLean (1980). Ce résultat suggère aussi que les pulvérisations d'insecticides ont été assez efficaces pour conserver une biomasse foliaire suffisante pour permettre aux arbres des étages dominants et codominants de réagir positivement aux conditions de croissance plus favorables.

Les intensités relativement faibles des éclaircies n'ont pas produit de grands écarts de volume marchand en 1968 (figure 1B). Quoique non significatif, l'écart de $20 \mathrm{~m}^{3} /$ ha entre les 


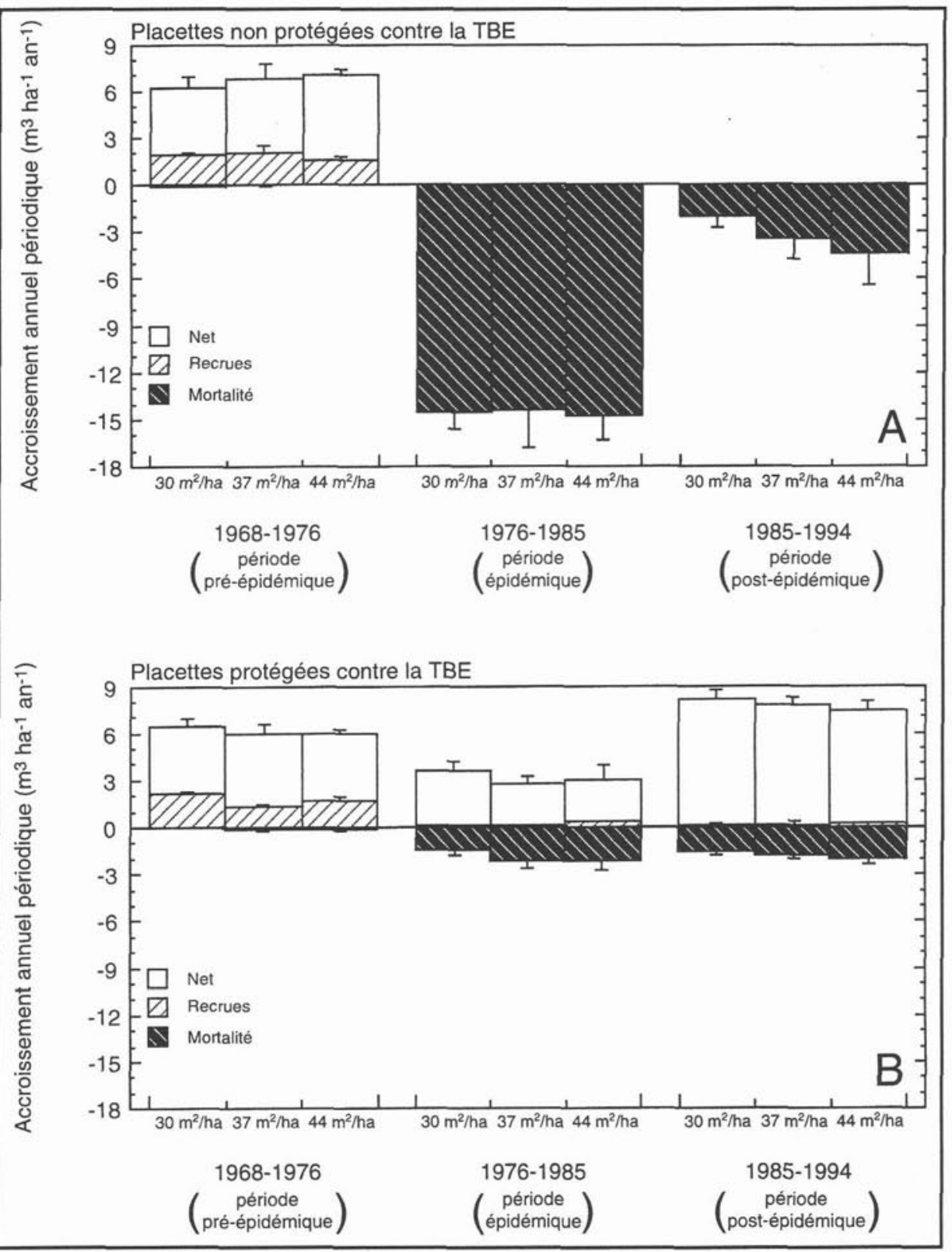

Figure 2. Accroissement annuel périodique en volume marchand des placettes regroupées en trois classes de surface terrière résiduelle pour un secteur non protégé contre la TBÉ (A) et un autre ayant bénéficié de pulvérisations aériennes annuelles d'insecticides entre 1978 et $1982(\mathrm{~B})$. placettes ayant une surface terrière résiduelle de $30 \mathrm{~m}^{2} / \mathrm{ha}$ et celles ayant 37 et $44 \mathrm{~m}^{2} / \mathrm{ha}$ a été peu à peu réduit au fil des ans de sorte qu'il n'était plus apparent à partir de 1989 (figure 1B). Ainsi, la production nette en volume marchand des placettes les plus éclaircies a eu tendance à être plus élevée que les deux autres niveaux de prélèvement même si aucune différence significative $(\alpha=0,05)$ n'a été détectée (figure 2B). Ce léger avantage de l'éclaircie la plus forte peut s'expliquer par un plus faible taux de mortalité des arbres de dimensions marchandes qui semble donc constituer l'essentiel du gain en volume associé à l'éclaircie (Reukema et Bruce 1977).

La ressemblance entre les volumes marchands observés en 1994 pour les trois niveaux de surface terrière résiduelle (figure 1B) appuie les observations démontrant qu'à long terme, l'intensité des éclaircies n'a que peu d'effet sur la production marchande totale des peuplements même en tenant compte des récoltes intermédiaires (Moller 1947; Pardé 1964;
Hamilton 1976; Hamilton 1981; Petterson 1993). Toutefois, l'accroissement annuel moyen en DHP des placettes a augmenté parallèlement à une diminution de la surface terrière totale résiduelle (figure 3). Ainsi, cette éclaircie par le bas a eu davantage d'impact sur le volume des tiges individuelles que sur le volume total d'un peuplement comme l'avaient déjà constaté Hatcher (1961), Matte (1962) et Lafond (1964) dans des sapinières québécoises. Puisque l'éclaircie par le bas favorise davantage l'accroissement des arbres dominants et codominants (Bertrand et Bolghari 1970; Bolghari 1980; Oliver et Murray 1983), les plus grandes dimensions des arbres éclaircis peuvent faire en sorte de devancer la récolte finale du peuplement ou d'augmenter le volume apte à produire du sciage. Par conséquent, un programme d'éclaircie par le bas ne semble justifié que dans le cas de peuplements destinés à la production de bois d'oeuvre.

À partir des informations recueillies dans les placettes échantillons et dans la littérature, il a été possible de superposer 


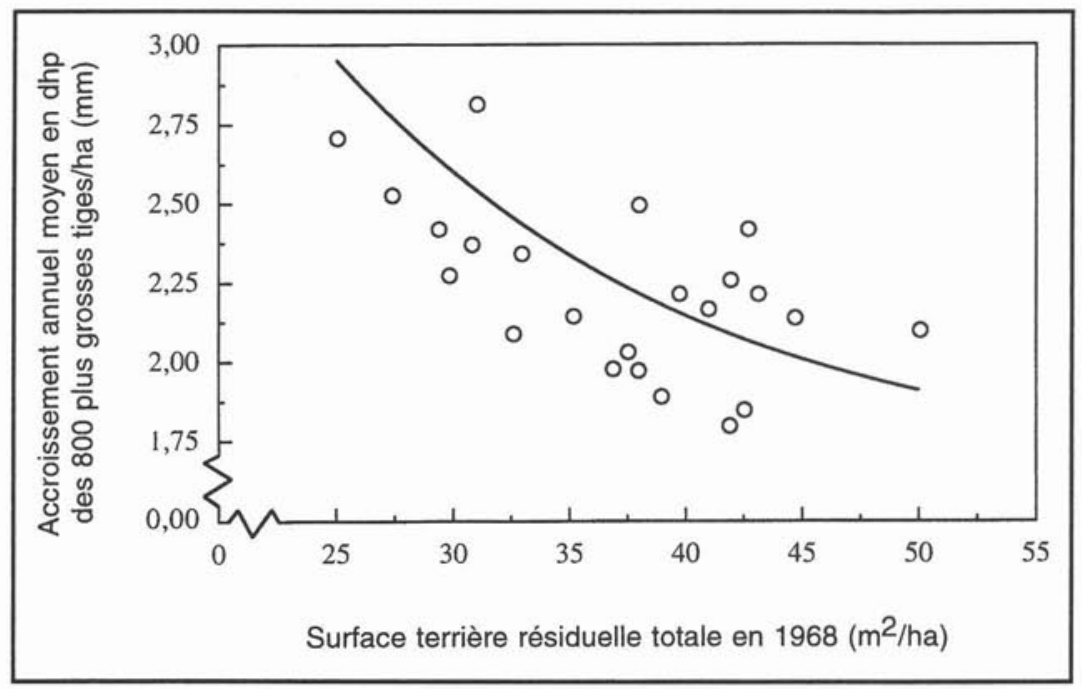

Figure 3. Accroissement annuel moyen en DHP des 800 plus grosses tiges/ha en fonction de la surface terrière totale résiduelle pour les placettes ayant bénéficié de pulvérisations aériennes annuelles d'insecticides entre 1978 et 1982.

Figure 4. Évolution du nombre d'arbres à l'hectare en fonction du volume total moyen par arbre pour les placettes ayant bénéficié de pulvérisations aériennes annuelles d'insecticides entre 1978 et 1982 . Les informations complémentaires $\left(\mathrm{DHP}_{\mathrm{q}}, \mathrm{H}_{\mathrm{d}}\right.$ et $\left.\rho_{\mathrm{r}}\right)$ ont été superposées selon la méthode décrite dans le texte. Chaque symbole associé aux trois regroupements de surface terrière totale résiduelle représente une année de mesurage.

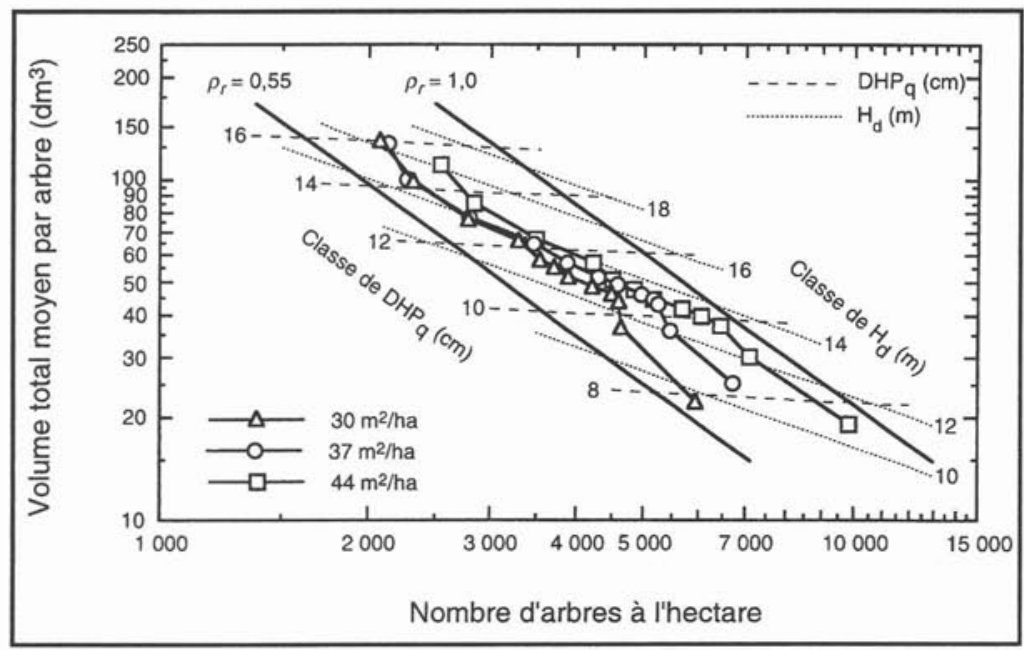

l'évolution du nombre total de tiges à un diagramme de gestion de la densité des peuplements (figure 4). L'utilisation de ce diagramme est toutefois limitée au présent secteur d'étude parce que les isolignes de hauteur et de diamètre ont été calculées uniquement à partir des placettes de ce dispositif expérimental. La figure 4 révèle que malgré la diminution du nombre d'arbres causée par les éclaircies, le cheminement (1968 à 1994) de l'ensemble des placettes est demeuré dans la zone de mortalité imminente induite par la compétition $\left(0,55<\rho_{\mathrm{r}}\right.$ $<1,0$ ) définie par Tremblay et al. (1997). Cette zone correspond à une densité de tiges tellement élevée en regard de leurs dimensions que certaines d'entre elles sont sujettes à mourir à un rythme s'approchant de la relation d'auto-éclaircie. Puisque les valeurs initiales du nombre de tiges à l'hectare des placettes les plus éclaircies étaient à l'intérieur de cette zone de mortalité imminente, il semble que l'intensité des éclaircies n'ait pas été assez forte pour minimiser la mortalité naturelle. La faible intensité des éclaircies de ce dispositif expérimental était justifiée par une prudence acquise à la suite d'observations de chablis importants dans des sapinières trop éclaircies (Hatcher 1961; Corriveau 1971). La figure 4 révèle cependant que le peuplement aurait pu profiter d'une éclaircie plus forte ou d'éclaircies subséquentes pour optimiser la taille des arbres à maturité.

L'évolution du nombre de tiges à l'hectare (figure 4) montre que le taux de mortalité était semblable à la relation d'auto-éclaircie (équation 1) pour toutes les densités initiales des placettes pendant la période 1968-1976. Cependant, pendant la période d'épidémie sévère de TBÉ (1976-1985), le taux de mortalité des placettes a été plus élevé que celui décrit par la relation d'auto-éclaircie, ce qui montre l'impact de défoliations sévères. Cet effet de l'épidémie de TBÉ a réduit l'écart de densité entre les deux plus bas niveaux de surface terrière résiduelle de sorte que leur cheminement a été semblable à partir de 1985 (figure 4). Après la période épidémique, le taux de mortalité est revenu aux valeurs pré-épidémiques, ce qui suggère que le volume des arbres morts pendant la période 1985 1994 (figure 2A) n'est pas attribuable aux seuls effets tardifs de l'épidémie de TBÉ mais aussi à la mortalité naturelle due à la compétition.

Malgré leur faible intensité, les éclaircies par le bas ont augmenté le diamètre moyen de l'ensemble des tiges (figure 4). 
Ainsi, à la dernière prise de mesure (1994), le diamètre quadratique des placettes les plus éclaircies ( 30 et $37 \mathrm{~m}^{2} /$ ha de surface terrière résiduelle) était de $16 \mathrm{~cm}$ comparativement à 15 $\mathrm{cm}$ pour les arbres des placettes les plus denses $\left(44 \mathrm{~m}^{2} / \mathrm{ha} \mathrm{de}\right.$ surface terrière résiduelle). Cet écart se reflète sur le volume moyen des arbres qui était d'environ $135 \mathrm{dm}^{3}$ pour les arbres des placettes les plus éclaircies et de $110 \mathrm{dm}^{3}$ pour les arbres des placettes plus denses. La figure 4 indique aussi que la hauteur dominante des placettes n'a pas été affectée par les traitements de sorte que les trois regroupements de surface terrière résiduelle étaient tous situés près de l'isoligne de $16 \mathrm{~m}$ en 1994. Puisque la croissance en hauteur des arbres n'est pratiquement pas affectée par l'intensité d'une éclaircie pour une large gamme de densité de peuplement (Schantz-Hansen 1956; Cayford 1964; Stiell 1980; Oliver et Murray 1983; Lanner 1985), il est possible d'utiliser cette caractéristique pour déduire le volume moyen des arbres d'un peuplement dont la densité aurait été davantage réduite que celle des placettes du présent dispositif. Ainsi, en prolongeant les isolignes de hauteur dominante de la figure 4 , on peut estimer qu'un hypothétique peuplement ayant une densité de 1000 tiges/ha et une hauteur dominante de $16 \mathrm{~m}$ serait composé de sapins de volume unitaire moyen de $215 \mathrm{dm}^{3}$. Ces dimensions pourraient être atteintes si un programme d'éclaircies avait maintenu la densité relative du peuplement légèrement en deçà de la limite inférieure de la zone de mortalité imminente induite par la compétition, c'est-à-dire à une valeur de $\rho_{\mathrm{r}}$ avoisinant 0,5 . Étant donné les risques de chablis inhérents à l'application d'une éclaircie dans ce type de peuplement, le maintien de la densité relative à une valeur conservatrice de 0,5 semble provisoirement constituer un objectif sylvicole à viser en attendant les résultats d'expériences qui auront testé une plus large gamme d'intensités de prélèvement.

\section{Remerciements}

L'amorce de ce projet de recherche et son suivi ont été assurés par Monsieur Hassanali Bolghari qui y a consacré beaucoup de temps et d'énergie jusqu'à son décès survenu en 1992. Des remerciements s'adressent à Messieurs Gilles Audy, Pascal Gagon, et Denis Thibault qui ont collaboré à la cueillette de données au fil des ans. Messieurs Jean-Martin Lussier, Philippe Meek, Jean Bégin, René Doucet et Fabien Caron ont aimablement révisé une version préliminaire du manuscrit et leurs commentaires ont été grandement appréciés.

\section{Bibliographie}

Aussenac, G. et A. Granier 1988. Effects of thinning on water stress and growth in Douglas-fir. Can. J. For. Res. 18: 100-105.

Aussenac, G., A. Granier et R. Naud 1982. Influence d'une éclaircie sur la croissance et le bilan hydrique d'un jeune peuplement de Douglas (Pseudotsuga menziesii (Mirb.) Franco). Can. J. For. Res. 12: 222-231.

Bailly, A. 1992. Éclaircir les peuplements résineux: un impératif écologique! Afocel-Armef. Informations-Forêt $n^{\circ} 4$.

Beaulieu, J. et Y. J. Hardy 1982. Mortalité du sapin baumier défolié par la tordeuse des bourgeons de l'épinette dans la région de la Gatineau au Québec. For. Chron. 58: 213-221.

Bertrand, V. et H. Bolghari 1970. L'effet d'une coupe d'éclaircie dans un peuplement dense d'épinettes et de sapin baumier âgé de 45 ans au sud-est du Québec. Gouv. du Québec, Min. Terres et Forêts, Serv. Rech, Mémoire ${ }^{\circ} 1$.

Bolghari, H. A. 1980. Croissance d'un peuplement de sapin-épinette à la suite de l'éclaircie et de la fertilisation au sud-est du Québec. Nat.
Can. 107: 135-149.

Brand, D. G. 1986. Competition-induced changes in developmental features of planted Douglas-fir in southwestern British Columbia. Can. J. For. Res. 16: 191-196.

Brix, H. 1983. Effects of thinning and nitrogen fertilization on growth of Douglas-fir: relative contribution of foliage quantity and efficiency. Can. J. For. Res. 13: 167-175.

Cayford, J. H. 1964. Reproduction and residual stand development following cutting in red pine-jack pine stands in Manitoba. Can. Dept. For., For. Res. Branch., Dept. For. Pub. 1010.

Corriveau, A. 1971. L'intensité de l'éclaircie, ses effets sur un peuplement de sapin baumier de 40 ans. Min. Envir., Serv. Can. For., Cent. Rech. For. Lau., Rap. Inf. Q-F-X-20.

Coyea, M. R. et H. A. Margolis 1994. The historical reconstruction of growth efficiency and its relationship to tree mortality in balsam fir ecosystems affected by spruce budworm. Can. J. For. Res. 24: 2208-2221.

Davis, L. S. et K. N. Johnson 1987. Forest management, 3rd edition. McGraw-Hill Book Company, New York, USA.

Drew, T. J. et J. W. Flewelling 1979. Stand density management: an alternative approach and its application to Douglas-fir plantations. For. Sci. 25: 518-532.

Guitton, J.-L. et F. Ruchaud 1996. Conséquences écologiques de l'éclaircie des peuplements résineux. Afocel-Armef, InformationsForêt, Fiche n_523.

Hamilton, G. J. 1976. The Bowmont Norway spruce thinning experiment 1930-1974. Forestry 49: 109-121.

Hamilton, G. J. 1981. The effects of high intensity thinning on yield. Forestry 54: 1-15.

Harrington, C. A. et D. L. Reukema 1983. Initial shock and longterm stand development following thinning in a Douglas-fir plantation. For. Sci. 29: 33- 46 .

Hatcher, R. J. 1961. Partial cutting balsam fir stands on the Epaule river watershed, Quebec. Can. Dept. of Forestry, For. Res. Branch., Tech. Note 105.

Honer, T. G., M. F. Ker et I. S. Alemdag 1983. Metric timber tables for the commercial tree species of central and eastern Canada. Can. For. Serv., Maritimes For. Res. Cent., Inf. Rep. M-X-140.

Lafond, A. 1964. Partial cutting in balsam fir stands. Pulp Pap. Mag. Can., Woodlands Review 65: $444-449$.

Lanner, R. M. 1985. On the insensitivity of height growth to spacing. For. Ecol. Manage. 13: 143-148.

MacLean, D. A. 1980. Vulnerability of fir-spruce stands during uncontrolled spruce budworm outbreaks: a review and discussion. For. Chron. 56: 213-221.

MacLean, D. A. et H. Piene 1995. Spatial and temporal patterns of balsam fir mortality in spaced and unspaced stands caused by spruce budworm defoliation. Can. J. For. Res. 25 : 902-911.

Matte, F. 1962. The first ten years of the Epaule project. Can. Pulp Pap. Ass., Woodlands Section Index n ${ }^{\circ} 2128$ (F-2).

Mitchell, R. G., R. H. Waring et G. B. Pitman 1983. Thinning lodgepole pine increases tree vigor and resistance to mountain pine beetle. For. Sci. 29: 204-211.

Moller, C. M. 1947. The effect of thinning, age, and site on foliage, increment, and loss of dry matter. J. For. 45: 393-404.

Newton, P. F. et G. F. Weetman 1993. Stand density management diagrams and their development and utility in black spruce management. For. Chron. 69: 421-430.

Oliver, C. D. et M. D. Murray 1983. Stand structure, thinning prescriptions, and density indexes in a Douglas-fir thinning study, Western Washington, U.S.A. Can. J. For. Res. 13: 126-136.

Pardé, J. 1964. Intensité des éclaircies et production ligneuse. Rev. For. Fran. 16: 936-945.

Pardé, J. et J. Bouchon 1988. Dendrométrie, $2^{\mathrm{e}}$ édition. ENGREF, Nancy, France.

Perron, J.-Y. 1985. Tarif de cubage — volume marchand brut. Gouvernement du Québec, Ministère de l'Énergie et des Ressources. 
Petterson, N. 1993. The effect of density after precommercial thinning on volume and structure in Pinus sylvestris and Picea abies stands. Scan. J. For. Res. 8: 528-539.

Piene, H. 1978. Effects of increased spacing on carbon mineralization rates and temperature in a stand of young balsam fir. Can. J. For. Res. 8: $398-406$.

Piene, H. 1989. Spruce budworm defoliation and growth loss in young balsam fir: defoliation in spaced and unspaced stands and individual tree survival. Can. J. For. Res. 19: 1211-1217.

Pothier, D. et H. A. Margolis 1991. Analysis of growth and light interception of balsam fir and white birch saplings following precommercial thinning. Ann. Sci. For. 48: 123-132.

Potvin, C., M.J. Lechowicz et S. Tardif 1990. The statistical analysis of ecophysiological response curves obtained from experiments involving repeated measures. Ecology $71: 1389-1400$.

Reukema, D. L. et D. Bruce 1977. Effects of thinning on yield of Douglas-fir: concepts and some estimates obtained by simulation. USDA For. Serv., Pacific Northwest For. Rang. Exp. Stn., Gen. Tech. Rep. PNW-58.

Rondeux, J. 1993. La mesure des arbres et des peuplements forestiers. Presses Agronomiques de Gembloux, Gembloux, Belgique. Schantz-Hansen, T. 1956. Twenty-five-year summary of red pine thinning plots: Cloquet experimental forest. J. For. 54: 512-514.
Stiell, W. M. 1980. Response of white spruce plantation to three levels of thinning from below, 1958-1978. For. Chron. 56: 21-27.

Thibault, D., J. Bégin et L. Bélanger 1995. Relations entre les indicateurs de croissance du sapin baumier en début d'épidémie et sa vulnérabilité à la tordeuse des bourgeons de l'épinette. Can. J. For. Res. 25: 1292-1302.

Tremblay, S., J. Bégin et L. Bélanger 1997. Élaboration d'un guide de gestion de la densité pour le sapin baumier, l'épinette noire et le pin gris. Rapport d'activités remis au Ministère des Ressources naturelles du Québec, Faculté de foresterie et de géomatique, Université Laval, Sainte-Foy, Québec.

Vézina, P.-É. et A. Linteau 1968. Growth and yield of balsam fir and black spruce in Québec. For. Res. Lab., Québec Region., Inf. Rep. Q-X-2.

Weetman, G. F. 1975. Ten-year growth response of black spruce to thinning and fertilization treatments. Can. J. For. Res. 5: 302-309. Weetman, G. F., M. R. Roberge et C. H. Meng 1980. Black spruce: 15 -year growth and microbiological responses to thinning and fertilization. Can. J. For. Res. 10: 502-509.

Whitehead, D., P. G. Jarvis et R. H. Waring 1984. Stomatal conductance, transpiration, and resistance to water uptake in a Pinus sylvestris spacing experiment. Can. J. For. Res. 14: 692-700. 